\title{
Phenomenological description of electromagnetic and strong interactions in rotating frames at collisions of high energy nuclei
}

\author{
Alexander J. Silenko* \\ Research Institute for Nuclear Problems, Belarusian State University, Minsk, Belarus, \\ Bogoliubov Laboratory of Theoretical Physics, Joint Institute for Nuclear Research, Dubna, \\ Russia \\ E-mail: alsilenko@mail.ru

\section{Oleg V. Teryaev} \\ Bogoliubov Laboratory of Theoretical Physics, Joint Institute for Nuclear Research, Dubna, \\ Russia \\ E-mail: teryaevetheor.jinr.ru
}

\begin{abstract}
Interaction of quarks in rotating frames described by the vector and scalar Cornell potentials is considered. The vector potential is defined similarly to the electromagnetic potential. The derived relativistic Hamiltonian in the Foldy-Wouthuysen representation is exact for terms of the zeroth and first orders in the Planck constant and for such terms of the second order which describe contact interactions. The classical limit of this Hamiltonian is also exact for terms of the zeroth and first orders in the Planck constant and is determined for the first time. The electromagnetic interaction is taken into account. General expressions for the angular velocity of spin precession are derived for both the vector and scalar Cornell potentials. For the both potentials, the dependence of the angular velocity of spin precession on the force acting on the quark is calculated. This force can be in principle determined with an analysis of experimental data. The energy of the spin-orbit interaction is rather high (of the order of $100 \mathrm{MeV}$ ).
\end{abstract}

XXII International Baldin Seminar on High Energy Physics Problems,

15-20 September 2014

JINR, Dubna, Russia

${ }^{*}$ Speaker. 


\section{Introduction}

Peripheral collisions of heavy ions generate global or local rotations of nuclear matter which are characterized by an angular velocity normal to the reaction plane. An appearance of vorticity of nuclear matter attracts significant interest (see Ref. [1] and references therein). It is naturally to suppose an existence of connection of this phenomenon with spin effects, in particular, with a quark polarization up or down relative to the reaction plane. We use the conventional Cornell potential for a phenomenological description of spin effects. It can be included into the Dirac equation in two possible ways. It is the most natural to consider the quark interaction like an electromagnetic interaction because the quanta of these interactions, photon and gluon, has the same spin. In this case, the Cornell potential is included into the four-potential of the Dirac equation. Another possibility is an inclusion of the Cornell potential into the scalar potential [2]. One often realizes the both possibilities and introduces the Cornell potential in the two (vector and scalar) forms $[3,4,5]$. Certainly, the vector Cornell potential is a four-vector. The use of traditional quantum-mechanical approaches allows one to solve the resulting equation only in some specific case, when the parameters of the scalar and vector potentials are very specific [3, 4, 5]. In all above-mentioned references, the Dirac equation in the Minkowski spacetime was considered.

In the present work, we use the both scalar and vector Cornell potentials and solve the more general problem of a strongly interacting Dirac particle (quark) in a rotating frame. The use of the covariant Dirac equation in a rotating frame instead of the usual Dirac equation in the Minkowski spacetime seems us to be a perspective way to a description of nuclear matter under peripheral collisions of heavy ions. In this case, tho total angular momentum can be rather large. Unlike other investigators, we use the approach based on the relativistic Foldy-Wouthuysen (FW) transformation and find a general solution of the problem for arbitrary parameters of the scalar and vector potentials. A possibility to restrict ourselves to a consideration of local interactions (because of short-range nuclear forces) allows us to realize advantages of this approach. Recently developed methods of the relativistic FW transformation $[6,7,8]$ (see also Ref. [9, 10, 11] and references therein) ensure fulfilling this transformation in arbitrarily strong external fields. While this approach has not been used for strong interactions, it has proven itself as a powerful tool for a description of electromagnetic and gravitational interactions of single particles $[9,10]$.

We use the system of units $\hbar=1, c=1$. Nevertheless, we include the Planck constant into several formulas. The world indices are labeled by Greek letters, whereas we reserve Latin letters from the beginning of the alphabet for tetrad indices. The Latin letters $i, j, k \ldots$ denote spatial world indices.

\section{Electromagnetic interactions of a Dirac particle in a rotating frame}

The initial covariant Dirac equation has the form (see Ref. [12] and references therein)

$$
\left(i \hbar \gamma^{a} D_{a}-m\right) \Psi=0, \quad a=0,1,2,3,
$$

where $D_{a}$ is the spinor covariant derivative. The Dirac matrices $\gamma^{a}$ are defined in local Lorentz frames and have the usual form. Equation (2.1) describes gravitational (inertial) and electromagnetical interactions. 
In Ref. [12], an exact Hermitian Hamiltonian in the Dirac representation corresponding to Eq. (2.1) has been determined. To give a more detailed description of electromagnetic interactions of a Dirac particle, an anomalous magnetic moment (AMM) of the particle should be taken into account. For this purpose, we use the generalization [13] of the initial equation, introducing the $\mathrm{AMM} \mu^{\prime}$ and the electric dipole moment (EDM) $d$ :

$$
\left(i \hbar \gamma^{a} D_{a}-m+\frac{\mu^{\prime}}{2} \sigma^{a b} F_{a b}+\frac{d}{2} \sigma^{a b} G_{a b}\right) \Psi=0 .
$$

The use of tetrad indices for Dirac matrices is caused by the definition of the three-component spin (pseudo)vector in the particle rest frame. This frame belongs to local Lorentz frames. After passing to a Minkowski spacetime, Eq. (2.2) coincides with the corresponding equation for a Dirac particle with the AMM and EDM (see Ref. [14]). Quantities $F_{a b}$ and $G_{a b}$ are defined by $F_{a b}=e_{a}^{\mu} e_{b}^{v} F_{\mu \nu}$ and $G_{a b}=e_{a}^{\mu} e_{b}^{v} G_{\mu \nu}$, where $F_{\mu \nu}=(\boldsymbol{E}, \boldsymbol{B})$ is the electromagnetic field tensor and the tensor $G_{\mu \nu}=(-\boldsymbol{B}, \boldsymbol{E})$ is obtained with the dual transformation $\left(G^{\mu v}=\frac{1}{2} \eta^{\mu \nu \lambda \rho} F_{\lambda \rho}\right)$. We can mention that $F_{a b}=(\mathfrak{E}, \mathfrak{B}), G_{a b}=(-\mathfrak{B}, \mathfrak{E})$, where

$$
\begin{gathered}
\mathfrak{E}=\boldsymbol{E}+\boldsymbol{G}, \quad \mathfrak{B}=\boldsymbol{B}, \\
\boldsymbol{G}=\boldsymbol{B} \times(\boldsymbol{\omega} \times \boldsymbol{r}), \quad \boldsymbol{E}=-\nabla \Phi-\frac{\partial \boldsymbol{A}}{\partial t}, \quad \boldsymbol{B}=\nabla \times \boldsymbol{A} .
\end{gathered}
$$

The quantities $\mathfrak{E}$ and $\mathfrak{B}$ are the effective fields in the rotating frame.

The general form of the Dirac Hamiltonian found in Ref. [12] does not contain additional terms characterizing the AMM and EDM. In the considered case, an inclusion of these terms lead to the following Dirac Hamiltonian [13]:

$$
\mathscr{H}=\beta m+e \Phi+\boldsymbol{\alpha} \cdot \boldsymbol{\pi}-\boldsymbol{\omega} \cdot(\boldsymbol{r} \times \boldsymbol{\pi})-\frac{\hbar}{2} \boldsymbol{\omega} \cdot \boldsymbol{\Sigma}-\boldsymbol{\Pi} \cdot \mathscr{M}+i \boldsymbol{\gamma} \cdot \mathscr{P},
$$

where

$$
\mathscr{M}=\mu^{\prime} \mathfrak{B}+d \mathfrak{E}, \quad \mathscr{P}=\mu^{\prime} \mathfrak{E}-d \mathfrak{B}, \quad \boldsymbol{\pi}=-i \hbar \nabla-e \boldsymbol{A} .
$$

Now we fulfil the transformation to the FW representation [15] with the method developed in Refs. [7, 8]. This representation holds a special place in quantum mechanics due to some unique properties. In this representation, quantum mechanical operators for relativistic particles in an external field have the same form as in the nonrelativistic quantum theory. In particular, the position operator [16] and momentum operator are equal to $\boldsymbol{r}$ and $\boldsymbol{p}=-i \hbar \nabla$, and the polarization operator for spin-1/2 particles is expressed by the Dirac matrix $\Pi$. In other representations, these operators are expressed by much more cumbersome formulas (see Refs. [6, 15]). The relations between the operators in the FW representation are analogous to the relations between the corresponding classical quantities. The simple form of operators corresponding to classical observables is a great advantage of this representation. The above-mentioned properties of the FW representation allow using it successfully for passing to the semiclassical approximation and to the classical limit of relativistic quantum mechanics $[15,17]$. We note that the Hamiltonian and all other operators are diagonal in two spinors (block-diagonal) in this representation. 
When the FW representation is used, the passage to the classical limit is usually accomplished by simply replacing the operators in the expressions for the Hamiltonian and in the operator equations of motion with the corresponding classical quantities. The possibility of such a replacement, explicitly or implicitly used in practically all works devoted to the relativistic FW transformation, was recently rigorously proved in Ref. [9]. This possibility radically simplifies interpreting the basic quantum mechanics equations, especially in the relativistic case.

Initial Hamiltonian (2.5) can be presented in the following general form:

$$
\mathscr{H}=\beta m+\mathscr{E}+\mathscr{O}, \quad \beta \mathscr{E}=\mathscr{E} \beta, \quad \beta \mathscr{O}=-\mathscr{O} \beta,
$$

where $\mathscr{E}$ and $\mathscr{O}$ are even and odd (diagonal and off-diagonal in two spinors) operators, respectively.

The Hamiltonian transformed to the FW representation is exact for terms proportional to the zeroth and first powers of the Planck constant and also for terms describing contact interactions and proportional to $\hbar^{2}$. It is given by $[7,10]$

$$
\begin{gathered}
\mathscr{H}_{F W}=\beta \varepsilon+\mathscr{E}-\frac{1}{8}\left\{\frac{1}{\varepsilon(\varepsilon+m)},[\mathscr{O},[\mathscr{O}, \mathscr{F}]]\right\}, \\
\mathscr{F}=\mathscr{E}-i \frac{\partial}{\partial t}, \quad \varepsilon=\sqrt{m^{2}+\mathscr{O}^{2}} .
\end{gathered}
$$

In the considered case, it is convenient to present this Hamiltonian in the form [13]

$$
\begin{gathered}
\mathscr{H}_{F W}=\mathscr{H}_{F W}^{(D)}+\mathscr{H}_{F W}^{(\text {add })}, \\
\mathscr{H}_{F W}^{(D)}=\beta \varepsilon^{\prime}+e \Phi-\boldsymbol{\omega} \cdot(\boldsymbol{r} \times \boldsymbol{\pi})-\frac{\hbar}{2} \boldsymbol{\omega} \cdot \boldsymbol{\Sigma}-\frac{e \hbar}{4}\left\{\frac{1}{\varepsilon^{\prime}}, \boldsymbol{\Pi} \cdot \boldsymbol{B}\right\} \\
+\frac{e \hbar}{8}\left\{\frac{1}{\varepsilon^{\prime}\left(\varepsilon^{\prime}+m\right)},[\boldsymbol{\Sigma} \cdot(\boldsymbol{\pi} \times \mathfrak{E}-\mathfrak{E} \times \boldsymbol{\pi})-\hbar \nabla \cdot \mathfrak{E}]\right\}, \\
\mathscr{H}_{F W}^{(a d d)}=\frac{1}{4}\left\{\frac{1}{\varepsilon^{\prime}},[\boldsymbol{\Sigma} \cdot(\boldsymbol{\pi} \times \mathscr{P}-\mathscr{P} \times \boldsymbol{\pi})-\hbar \nabla \cdot \mathscr{P}]\right\}-\boldsymbol{\Pi} \cdot \mathscr{M} \\
+\frac{1}{4}\left\{\frac{1}{\varepsilon^{\prime}\left(\varepsilon^{\prime}+m\right)},[(\boldsymbol{\Pi} \cdot \boldsymbol{\pi})(\boldsymbol{\pi} \cdot \mathscr{M})+(\mathscr{M} \cdot \boldsymbol{\pi})(\boldsymbol{\Pi} \cdot \boldsymbol{\pi})\right. \\
\left.\left.+2 \pi \beta \hbar(\boldsymbol{\pi} \cdot \mathscr{J}+\mathscr{J} \cdot \boldsymbol{\pi})+\beta \frac{\hbar}{2}\{([\boldsymbol{\omega} \times \boldsymbol{r}] \cdot \nabla),(\boldsymbol{\pi} \cdot \mathscr{P})\}\right]\right\},
\end{gathered}
$$

where $\varepsilon^{\prime}=\sqrt{m^{2}+\boldsymbol{\pi}^{2}}$ and $\mathscr{J}=[\nabla \times \mathscr{M}-\partial \mathscr{P} /(\partial t)] /(4 \pi)$. The operator $\mathscr{H}_{F W}^{(D)}$ results from a transformation of the Hamiltonian, corresponding Eq. (2.1), and the operator $\mathscr{H}_{F W}^{(\text {add })}$ contains terms, proportional to the AMM and EDM. In the last term of Eq. (2.11), the nabla operator acts on $\mathscr{P}$ and defines derivatives of this quantity.

It is important that Eqs. (2.9)-(2.11) are exact in relation to the above-mentioned terms proportional to the zeroth, first, and second powers of $\hbar$. We are to underline that $\mu^{\prime}, d, \mathscr{P}$, and $\mathscr{M}$ are proportional to $\hbar$.

\section{Effective fields acting on particle and spin}

The results obtained in the previous section allow us to determine effective fields acting on a particle and a spin. Let us use the simplest way of this determination. 
Let $\boldsymbol{r}$ be the radius-vector of a spinning particle possessing an EDM. Let us compare the spin dynamics in the frame rotating with the angular velocity $\boldsymbol{\omega}$ and in the instantly accompanying frame moving with the velocity $\boldsymbol{V}=\boldsymbol{\omega} \times \boldsymbol{r}$ relative to the lab frame. ${ }^{1}$ It is convenient to consider the simplest case when the particle velocity is equal to zero in the both frames in the considered moment of time. To describe the spin motion in the instantly accompanying frame, we suppose $\boldsymbol{\omega}=0$ in Eqs. (2.9)-(2.11). For a particle at rest, the spin-dependent terms in the two Hamiltonians are given by

$$
\mathscr{H}_{F W}^{(a c c)}=-\mu \boldsymbol{\Pi} \cdot \boldsymbol{B}^{\prime}-d \boldsymbol{\Pi} \cdot \boldsymbol{E}^{\prime}, \quad \mathscr{H}_{F W}^{(r o t)}=-\frac{\hbar}{2} \boldsymbol{\omega} \cdot \boldsymbol{\Sigma}-\mu \boldsymbol{\Pi} \cdot \mathfrak{B}-d \boldsymbol{\Pi} \cdot \mathfrak{E},
$$

where $\boldsymbol{E}^{\prime}$ and $\boldsymbol{B}^{\prime}$ are the field acting on the particle in the instantly accompanying frame.

In the general case, the spin-dependent terms in the FW Hamiltonian have the form $\boldsymbol{\Omega} \cdot \boldsymbol{s}$, where $\boldsymbol{\Omega}$ is the operator of the angular velocity of spin precession and $\boldsymbol{s}$ is the spin operator equal to $\hbar \Pi / 2$ or $\hbar \boldsymbol{\Sigma} / 2$. A difference between the angular velocities of spin precession in the two frames can be caused by (i) the frame rotation with the angular velocity $\boldsymbol{\omega}$ and (ii) the Thomas precession with the angular velocity $\boldsymbol{\omega}_{T}=-\left[\gamma^{2} /(\gamma+1)\right] \boldsymbol{\beta} \times \dot{\boldsymbol{\beta}}, \boldsymbol{\beta}=\boldsymbol{v} / c$. However, the Thomas precession is zero for the particle at rest. Therefore, Eq. (3.1) results in

$$
\mathfrak{E}=\boldsymbol{E}^{\prime}, \quad \mathfrak{B}=\boldsymbol{B}^{\prime}, \quad \boldsymbol{E}=\boldsymbol{E}^{\prime}-\boldsymbol{B}^{\prime} \times(\boldsymbol{\omega} \times \boldsymbol{r}) .
$$

These relations have a simple physical sense. Let us consider the Earth's rotating frame. In this case, the effective fields acting on the particle spin (but not on the particle momentum!) are equal to fields defined with formulas of special relativity for a given distribution of charges and currents.

To determine the particle motion, we are to find the classical limit of the Hamiltonian (2.9) and to calculate the force. This force is given by [18] $\boldsymbol{F}=d \mathfrak{P} /(d t)$, where $\mathfrak{P}^{i}=-\delta^{i j} p_{j}+g^{0 i} p_{0}$ is a spatial part of the covariant four-momentum $\mathfrak{P}^{\mu}=g^{\mu v} p_{v} \cdot{ }^{2}$ A sufficient precision is ensured by taking into account terms linear in $\boldsymbol{E}, \boldsymbol{B}$, and $\boldsymbol{\omega}$. After replacing of the operators in Eqs. (2.9)-(2.11) with corresponding classical quantities, we determine this force:

$$
\begin{gathered}
\boldsymbol{F}=\boldsymbol{E}+\boldsymbol{\beta} \times \boldsymbol{B}+\boldsymbol{F}_{C o r}+\boldsymbol{F}_{c f}, \quad \boldsymbol{F}_{C o r}=2 \boldsymbol{\pi} \times \boldsymbol{\omega}, \\
\boldsymbol{F}_{c f}=-\boldsymbol{\varepsilon}^{\prime} \boldsymbol{\omega} \times(\boldsymbol{\omega} \times \boldsymbol{r}),
\end{gathered}
$$

where $\boldsymbol{F}_{C o r}$ and $\boldsymbol{F}_{c f}$ are the Coriolis and centrifugal forces. Therefore, the effective electric field acting on the particle momentum is equal to $\boldsymbol{E}$. It differs from the effective electric field acting on the particle spin. The corresponding effective magnetic fields coincide.

\section{Phenomenological description of interaction of quarks with the Cornell potential}

\subsection{Cornell potential and its inclusion into the Dirac equation}

The standard method of phenomenological description of interaction of quarks is the use of the Cornell potential having the form

$$
V(r)=-\frac{a(r)}{r}+b(r) r
$$

\footnotetext{
${ }^{1}$ The results obtained in the present work can be applied for any inertial frame, in particular, for the center-of-mass one.

${ }^{2}$ Alternatively, the force can be defined as $-d \boldsymbol{p} /(d t)$, where $\boldsymbol{p}$ is a spatial part of $p_{v}$.
} 
In the first approximation, we can suppose that $a$ and $b$ are constant. In this case, $a \approx 100 \mathrm{MeV} \cdot \mathrm{fm}$, $b \approx 400 \mathrm{MeV} \cdot \mathrm{fm}^{-1}$. However, the second term in the Cornell potential is proportional to $r^{2}$ at condition that $r$ is small [19].

For a phenomenological description of interaction of quarks, the Cornell potential should be included into the Dirac equation. This can be made in different ways. There are some similarities between the strong and electromagnetic interactions of quarks. Therefore, an inclusion of the potential (4.1) into the four-vector potential $A^{\mu}=(\Phi, \boldsymbol{A})$ similarly to the Coulomb interaction is the most natural. In this case, $\Phi=\gamma \Phi_{0}, \boldsymbol{A}=\gamma \Phi_{0} \boldsymbol{V} / c, e \Phi_{0} \rightarrow V(r)$, where $\boldsymbol{V}$ is a velocity of the field source (quark) in a given frame. Such an approach allows us to introduce the normal and anomalous chromomagnetic moments of quarks. These moments are of the same order (see Ref. $[20,21])$.

Alternatively, the Cornell potential can be included into the Dirac equation as a scalar potential. In this case, the Cornell potential is multiplied by the matrix $\beta$, and its inclusion means the effective modification of the quark mass. If the electromagnetic interaction is not taken into account, the Dirac Hamiltonian takes the form

$$
\mathscr{H}=\beta[m+V(r)]+\boldsymbol{\alpha} \cdot \boldsymbol{p}-\boldsymbol{\omega} \cdot(\boldsymbol{r} \times \boldsymbol{p})-\frac{\hbar}{2} \boldsymbol{\omega} \cdot \boldsymbol{\Sigma}, \quad \boldsymbol{p}=-i \hbar \nabla .
$$

One mostly incorporated both the scalar and vector potentials into the Dirac equation $[3,4,5]$. For the second potential, the Cornell one replaced the scalar electromagnetic potential $\Phi$. The only case of an equality of the two incorporated potentials was investigated in Refs. [3, 4, 5]. This equality does not have any physical substantiation but it allows one to obtain exact solutions $[3,4$, 5]. In fact, the scalar and vector Cornell potentials present two different independent approaches to a description of the strong interaction. In Ref. [2], the vector potential was not used. In this work, the FW transformation was performed only for a nonrelativistic Dirac particle in the weak field approximation. In the present work, the much more general problem of a high-precision description of a relativistic quark in an arbitrarily strong field is solved thanks to the use of a more universal method of the FW transformation. This allows us to calculate the effect of the strong spin-orbit interaction of relativistic quarks.

The quantity $\omega$ is defined by the total angular momentum and the moment of inertia of colliding nuclei. Evidently, the assumption that the system of colliding nuclei rotates as a whole is approximate. It would be valid if the sum of the kinetic energies of the nuclei and the interaction energy were negative. However, this condition cannot be satisfied.

\subsection{Phenomenological description of strong interaction with the vector Cornell potential}

When the vector Cornell potential $\mathscr{A}^{\mu}=(\mathbb{F}, \mathscr{A})$ is used and the electromagnetic interaction of quarks is taken into account, the FW Hamiltonian takes the form (2.9)-(2.11). The potential $\mathbb{F}$ can be set equal to $V(r)$. The terms characterizing the EDM can be disregarded. This approach allows us to introduce the chromoelectric and chromomagnetic fields, $\mathscr{Q}$ and $\mathscr{R}$ :

$$
\mathscr{Q}=-\nabla \mathbb{F}-\frac{\partial \mathscr{A}}{\partial t}, \mathscr{R}=\nabla \times \mathscr{A} .
$$

We can take into account interactions conditioned by normal and anomalous chromomagnetic moments and also contact interactions. 
When one uses the assumption that the system of colliding nuclei rotates as a whole, the rotating frame can be applied. In this case, the centrifugal force should be counterbalanced on average by the attractive force acting between quarks.

While the rotating frame is convenient for a description of dynamics of quarks, the use of the lab frame is preferable for an analysis of observable effects. In this case, we should substitute $\boldsymbol{\omega}=0$. As a result, we obtain the following Hamiltonian:

$$
\begin{gathered}
\mathscr{H}_{F W}=\beta \varepsilon^{\prime}+e \Phi+\mathbb{F}+\frac{\hbar}{8 m^{2}}\left\{\frac{1}{\gamma(\gamma+1)},(\boldsymbol{\Sigma} \cdot[\boldsymbol{\pi} \times(e \boldsymbol{E}+\mathscr{Q})\right. \\
-(e \boldsymbol{E}+\mathscr{Q}) \times \boldsymbol{\pi}]-\hbar \nabla \cdot(e \boldsymbol{E}+\mathscr{Q}))\} \\
+\frac{\hbar}{8 m^{2}}\left\{\frac{1}{\gamma},\left(\boldsymbol { \Sigma } \cdot \left[\boldsymbol{\pi} \times\left(e G \boldsymbol{E}+G_{c} \mathscr{Q}\right)\right.\right.\right. \\
\left.\left.\left.-\left(e G \boldsymbol{E}+G_{c} \mathscr{Q}\right) \times \boldsymbol{\pi}\right]-\hbar \nabla \cdot\left(e G \boldsymbol{E}+G_{c} \mathscr{Q}\right)\right)\right\} \\
+\frac{\hbar}{4 m}\left\{\frac{1}{\gamma},(\boldsymbol{\Pi} \cdot[e \boldsymbol{B}+\mathscr{R}])\right\}-\frac{\hbar}{2 m}\left(\boldsymbol{\Pi} \cdot\left[e G \boldsymbol{B}+G_{c} \mathscr{R}\right]\right) \\
+G_{c}^{3}\left\{\frac{1}{\gamma(\gamma+1)},\left[\left(\left[e G \boldsymbol{B}+G_{c} \mathscr{R}\right] \cdot \boldsymbol{\pi}\right)(\boldsymbol{\Sigma} \cdot \boldsymbol{\pi})+(\boldsymbol{\Sigma} \cdot \boldsymbol{\pi})(\boldsymbol{\pi} \cdot[e G \boldsymbol{B}\right.\right. \\
\end{gathered}
$$

where $\gamma=\varepsilon^{\prime} / m=\sqrt{m^{2}+\boldsymbol{\pi}^{2}} / m$ is the Lorentz factor, $\boldsymbol{\pi}=\boldsymbol{p}-e \boldsymbol{A}-\mathscr{A}, G=(g-2) / 2, G_{c}=$ $\left(g_{c}-2\right) / 2, g_{c}$ is the chromomagnetic $g$ factor similar to the electromagnetic one, $\boldsymbol{j}=[\boldsymbol{\nabla} \times \boldsymbol{B}-$ $\partial \boldsymbol{E} /(\partial t)] /(4 \pi)$, and $\mathscr{I}=[\boldsymbol{\nabla} \times \mathscr{R}-\partial \mathscr{Q} /(\partial t)] /(4 \pi)$.

In the classical limit, the operator of the angular velocity of spin precession is given by

$$
\begin{aligned}
& \boldsymbol{\Omega}=\frac{1}{m c(\gamma+1)} \boldsymbol{\beta} \times(e \boldsymbol{E}+\mathscr{Q})+\frac{1}{m c} \boldsymbol{\beta} \times\left(e G \boldsymbol{E}+G_{c} \mathscr{Q}\right) \\
& -\frac{1}{m c \gamma}(e \boldsymbol{B}+\mathscr{R})-\frac{1}{m c}\left(e G \boldsymbol{B}+G_{c} \mathscr{R}\right) \\
& +\frac{\gamma}{m c(\gamma+1)}\left[\boldsymbol{\beta} \cdot\left(e G \boldsymbol{B}+G_{c} \mathscr{R}\right)\right] \boldsymbol{\beta} .
\end{aligned}
$$

The velocity of light $c$ is explicitly included into this equation. In this limit, the force is similar to the Lorentz force:

$$
\boldsymbol{F}=e \boldsymbol{E}+\mathscr{Q}+\boldsymbol{\beta} \times(e \boldsymbol{B}+\mathscr{R}) .
$$

Let $\mathscr{V}$ be the velocity of the quark which is the source of the strong and electromagnetic fields. If we neglect the spin-spin interaction of the quarks in the first approximation, we obtain the following relations

$$
\boldsymbol{B}=\frac{\mathscr{V}}{c} \times \boldsymbol{E}, \quad \mathscr{R}=\frac{\mathscr{V}}{c} \times \mathscr{Q}
$$

When the interacting quarks belong to different nuclei, the vectors $\boldsymbol{v}$ and $\mathscr{V}$ have opposite directions in the lab frame. At collisions of high energy nuclei, it can be often supposed that $|\boldsymbol{v}| \approx|\mathscr{V}| \approx c$. An opposite situation takes place when the vectors $\boldsymbol{v}$ and $\mathscr{V}$ are mainly co-directed. This situation can probably be realized in quark-gluon plasma due to a predominance of local 
interactions. For the stationarily rotating system of nuclei, the vector $\boldsymbol{v}$ is almost orthogonal to the vectors $\boldsymbol{B}$ and $\mathscr{R}$. In this case, the last term in Eq. (4.5) can be neglected in the first approximation.

Let us use the center-of-mass system and consider the simplest (but practically very important) case of a collision of two nucleons. In this case, $\mathscr{V}=-\boldsymbol{v}$. Let us determine the Hamiltonian of the first quark in the field of the second one. We restrict ourselves by a phenomenological description of main effects under peripheral collisions of relativistic nuclei. To perform a more precise quantitative analysis, one needs to take into account effects of retardation and to use the Breit equation as an initial one. Nevertheless, the approach used gives us a reasonable description of the force acting on the quark and of the torque acting on its spin.

When the electromagnetic interaction is neglected, the classical limit of the Hamiltonian (4.4) is given by

$$
H=\varepsilon^{\prime}+\mathbb{F}+\frac{1}{m c}\left(\frac{1}{\gamma}+\frac{1}{\gamma+1}+2 G_{c}\right) \boldsymbol{s} \cdot(\boldsymbol{\beta} \times \mathscr{Q}) .
$$

Here $s$ is the classical spin vector. In this approximation, the angular velocity of spin precession is equal to

$$
\boldsymbol{\Omega}=\frac{1}{m c}\left(\frac{1}{\gamma}+\frac{1}{\gamma+1}+2 G_{c}\right) \boldsymbol{\beta} \times \mathscr{Q}
$$

We take into account that the contribution of the strong interaction into the spin precession is predominant for any energy.

It is rather convenient to express the angular velocity of spin precession $\Omega$ in the lab frame (more precisely, in the center-of-mass one) in terms of the force (4.6) in the same frame. In the considered case, this force is given by

$$
\boldsymbol{F}=\left(2-\frac{1}{\gamma^{2}}\right)(e \boldsymbol{E}+\mathscr{Q})-\boldsymbol{\beta}(\boldsymbol{\beta} \cdot[e \boldsymbol{E}+\mathscr{Q}])
$$

When only the strong interaction is taken into account, the angular velocity of spin precession is therefore expressed as follows:

$$
\boldsymbol{\Omega}=\frac{1}{m c}\left(\frac{1}{\gamma}+\frac{1}{\gamma+1}+2 G_{c}\right) \frac{\boldsymbol{\gamma}^{2}}{2 \gamma^{2}-1} \boldsymbol{\beta} \times \boldsymbol{F} .
$$

Since the force $\boldsymbol{F}$ defines the change of the momentum, it can be in principle extracted from experimental data. Thus, Eq. (4.11) is convenient for an analysis of experimental effects.

The sign of the projection of $\boldsymbol{\Omega}$ onto the normal to the scattering plane depends on the sign of $G_{c}$. If $G_{c}<0$ (see Ref. [21]) and the Lorentz factor is large enough, the pseudovectors $\boldsymbol{\Omega}$ and $\boldsymbol{\omega}$ are oppositely directed.

Equation (4.11) defines the rather large angular velocity of spin precession which corresponds to the rather high energy of spin-orbit interaction (of the order of $100 \mathrm{MeV}$ ).

If one supposes that the system of nuclei rotates as a whole, $\boldsymbol{F}=-\boldsymbol{F}_{c f}$ and $\boldsymbol{v}=\boldsymbol{\omega} \times \boldsymbol{r}$.

The angular velocity of spin precession is of the same order of magnitude as the vorticity calculated in Ref. [1].

We can conclude that the strong interaction of quarks leads to the spin-orbit interaction which results in a spin rotation and in a preferable orientation of the spin. The latter effect is similar to a spin polarization in targets placed in a magnetic field. In the case of strange quarks, this effect can be an additional explanation of hyperon polarization [1]. 


\subsection{Phenomenological description of strong interaction with the scalar Cornell potential}

An interaction of quarks caused by the scalar Cornell potential can be determined in a similar way. We describe this interaction in a rotating frame and do not consider an electromagnetic interaction which is more weak. In principle, this interaction can be included into the initial Hamiltonian and the FW transformation of the Hamiltonian obtained can be carried out.

The interaction of quarks in the rotating frame characterized by the scalar Cornell potential is described by the Dirac Hamiltonian (4.2). The FW transformation of such Dirac Hamiltonians has been performed in Ref. [12]. With the use of Eqs. (2.24) and (3.1)-(3.6) from this work, we obtain the following relativistic Hamiltonian: ${ }^{3}$

$$
\begin{gathered}
\mathscr{H}_{F W}=\beta \varepsilon^{\prime \prime}-\boldsymbol{\omega} \cdot(\boldsymbol{r} \times \boldsymbol{p})-\frac{\hbar}{2} \boldsymbol{\omega} \cdot \boldsymbol{\Sigma} \\
+\beta \frac{\hbar}{4}\left\{\frac{1}{2 \varepsilon^{\prime \prime 2}+\left\{\boldsymbol{\varepsilon}^{\prime \prime},[m+V(r)]\right\}},[\boldsymbol{\Sigma} \cdot(\mathscr{E} \times \boldsymbol{p}-\boldsymbol{p} \times \mathscr{E})+\hbar \nabla \cdot \mathscr{E}]\right\}, \\
\varepsilon^{\prime \prime}=\sqrt{[m+V(r)]^{2}+\boldsymbol{p}^{2}}, \quad \mathscr{E}=-\nabla V(r)=-V^{\prime}(r) \frac{\boldsymbol{r}}{r} .
\end{gathered}
$$

Thus, the presence of the scalar Cornell potential also results in the spin-orbit interaction. In the considered case, the classical limit of the operator of the angular velocity of spin precession reads

$$
\boldsymbol{\Omega}=-\boldsymbol{\omega}+\frac{\mathscr{E} \times \boldsymbol{p}}{\varepsilon^{\prime \prime}\left[\varepsilon^{\prime \prime}+m+V(r)\right]} .
$$

As above, let us consider the quark interaction in the lab frame $($ when $\boldsymbol{\omega}=0)$ for a determination of observable spin effects. It is convenient to express the angular velocity of spin precession in terms of the force. In the classical limit, the force operator $\boldsymbol{F}=-\nabla \mathscr{H}_{F W}$ takes the form

$$
\boldsymbol{F}=\frac{[m+V(r)] \mathscr{E}}{\mathcal{\varepsilon}^{\prime \prime}}
$$

The velocity of the quark is given by

$$
v=\frac{p}{\varepsilon^{\prime \prime}}
$$

Thus, the Lorentz factor is equal to $\gamma=\varepsilon^{\prime \prime} /[m+V(r)]$.

These formulas make it possible to connect the angular velocity of spin precession with the force:

$$
\boldsymbol{\Omega}=-\frac{\gamma}{(\gamma+1)[m+V(r)] c} \boldsymbol{\beta} \times \boldsymbol{F} .
$$

The connection between the angular velocity of spin precession and the force is general and almost exact when the scalar Cornell potential is considered. In the case of the vector Cornell potential, the corresponding connection is approximate. The energy of the spin-orbit interaction defined by the scalar Cornell potential is also rather high (of the order of $100 \mathrm{MeV}$ ).

\section{Conclusions}

We considered the strong and electromagnetic interactions of Dirac particles (quarks) in rotating frames. We used the scalar and vector Cornell potentials for a phenomenological description

\footnotetext{
${ }^{3}$ In the above-mentioned equations, $\mathscr{F}=1, \boldsymbol{K}=-\boldsymbol{\omega} \times \boldsymbol{r} / c$, and $m c^{2} V$ should be substituted by $m+V(r)$.
} 
of the strong interaction. A large angular momentum of the system of colliding nuclei leads to the effective rotation. The vector Cornell potential is similar to the four-vector potential of the electromagnetic field. Since the strong interaction is much more close to the electromagnetic interaction than to the scalar one, the use of the vector Cornell potential is very natural. Unlike previous investigations $[2,3,4,5]$, we used the method of the relativistic FW transformation [6, 7] applicable in arbitrarily strong external fields and determined the relativistic FW Hamiltonian. This Hamiltonian is exact relative to terms of the zeroth and first orders in the Planck constant and such terms of the order of $\hbar^{2}$ which describe contact interactions. We found the exact classical limit of this Hamiltonian. The adequate and exact classical limit of the strong interaction of quarks characterized by the Cornell potential was determined for the first time. We also took into account the electromagnetic interaction which may not be negligible. Effective fields acting on the particle and spin were found. General formulas for the angular velocity of spin precession were derived. These formulas correspond to the high energy of the spin-orbit interaction (of the order of $100 \mathrm{MeV}$ ) for both the vector and scalar Cornell potentials. For these potentials, we determined the relations between the angular velocity of spin precession and the force acting on the quark. Since this force defines the change of the momentum, it can be in principle extracted from experimental data.

\section{Acknowledgments}

This work was supported in part by the Belarusian Republican Foundation for Fundamental Research (Grant No. $\Phi 14 \mathrm{D}-007$ ) and by the Heisenberg-Landau program of the German Ministry for Science and Technology (Bundesministerium für Bildung und Forschung).

\section{References}

[1] M. Baznat, K. Gudima, A. Sorin, O. Teryaev, Phys. Rev. C 88, 061901 (2013).

[2] U.D. Jentschura, J.H. Noble, J. Phys. A: Math. Theor. 47, 045402 (2014).

[3] H. Hassanabadi, E. Maghsoodi, S. Zarrinkamar, H. Rahimov, Adv. High Energy Phys. 2012, 707041 (2012); H. Hassanabadi, E. Maghsoodi, S. Zarrinkamar, Ann. Phys. (Berlin) 525, 944 (2013).

[4] M. Hamzavi, A.A. Rajabi, Ann. Phys. (New York) 334, 316 (2013); Chin. Phys. B 22, 090301 (2013); Chin. Phys. C 37, 103102 (2013); A.A. Rajabi, M. Hamzavi, Few-Body Syst. 54, 2067 (2013).

[5] L.A. Trevisan, C. Mirez, F.M. Andrade, Few-Body Syst. 55, 1055 (2014).

[6] A.J. Silenko, J. Math. Phys. 44, 2952 (2003).

[7] A.J. Silenko, Phys. Rev. A 77, 012116 (2008).

[8] A.J. Silenko, Phys. Rev. A 91, 022103 (2015).

[9] A.J. Silenko, Phys. Part. Nucl. Lett. 10, 91 (2013).

[10] A.J. Silenko, Theor. Math. Phys. 176, 987 (2013).

[11] A.J. Silenko, Phys. Rev. A 91, 012111 (2015).

[12] Yu.N. Obukhov, A.J. Silenko, O.V. Teryaev, Phys. Rev. D 84, 024025 (2011).

[13] Yu.N. Obukhov, A.J. Silenko, O.V. Teryaev, Phys. Part. Nucl. 45, 234 (2014). 
[14] A.J. Silenko, Russ. Phys. J. 48, 788 (2005).

[15] L.L. Foldy, S.A. Wouthuysen, Phys. Rev. 78, 29 (1950).

[16] T.D. Newton, E.P. Wigner, Rev. Mod. Phys. 21, 400 (1949).

[17] J.P. Costella, B.H.J. McKellar, Am. J. Phys. 63, 1119 (1995).

[18] L.D. Landau, E.M. Lifshitz, The Classical Theory of Fields, 3rd edn, Pergamon Press, Oxford 1973, p. 251 .

[19] Ya.Ya. Balitskii, Nucl. Phys. B 254, 166 (1983).

[20] D. Diakonov, Prog. Part. Nucl. Phys. 51, 173 (2003).

[21] N. Kochelev, N. Korchagin, Phys. Lett. B 729, 117 (2014). 